\title{
GENERATORS AND RELATIONS FOR THE SPECIAL LINEAR GROUP OVER A DIVISION RING'
}

\author{
SHERRY M. GREEN
}

\begin{abstract}
Let $R$ be a division ring, $n \geqslant 3$ an integer, and let $\operatorname{SL}(n, \Re)$ be the special linear group over $\Re$. In this paper a presentation in terms of generators and relations is given for $\operatorname{SL}(n, \Re)$.
\end{abstract}

Let $\Re$ be a division ring, $\Re^{*}$ its group of units. If $G$ is a group and $a, b \in G$ we write $(a, b)=a b a^{-1} b^{-1}$ for the commutator of $a$ and $b$ and $(G, G)$ for the commutator subgroup of $G$. If $n$ is an integer, let $\operatorname{GL}(n, \Re)$ denote the general linear group consisting of all $n \times n$ invertible matrices, and let $\operatorname{SL}(n, \Re)$ denote the special linear group consisting of all $n \times n$ matrices of determinant 1 (in the sense of Dieudonné). If $i$ and $j$ are distinct integers between 1 and $n$ and $u \in \Re$, let $e_{i j}(u) \in \operatorname{SL}(n, \Re)$ denote the matrix with entry $u$ in the $(i, j)$ th place, ones on the diagonal, and zeros elsewhere. It is well known that the $e_{i j}(u)$ generate $\operatorname{SL}(n, \Re)$.

If $u \in \mathscr{R}^{*}$ we define elements $m_{i j}(u)$ and $d_{i j}(u)$ of $\operatorname{SL}(n, \Re)$ to be

$$
m_{i j}(u)=e_{i j}(u) e_{j i}\left(-u^{-1}\right) e_{i j}(u)
$$

and

$$
d_{i j}(u)=m_{i j}(u) m_{i j}(-1)
$$

Note that $d_{i j}(u)=\operatorname{diag}\left(1, \ldots, 1, u, 1, \ldots, 1, u^{-1}, 1, \ldots, 1\right)$, the diagonal matrix with $u$ in the $(i, i)$ th place and $u^{-1}$ in the $(j, j)$ th place.

If $u, v \in \Re^{*}$ we define the element $a_{1}(u, v)$ of $\operatorname{SL}(n, \Re)$ to be

$$
a_{1}(u, v)=d_{12}(u) d_{12}(v) d_{12}(v u)^{-1} .
$$

Note that $a_{1}(u, v)=\operatorname{diag}((u, v), 1, \ldots, 1)$, the diagonal matrix with $(u, v)$ in the $(1,1)$ st place.

We will now give a description of generators and relations for $\operatorname{SL}(n, \Re)$.

THEOREM. If $n \geqslant 3, \operatorname{SL}(n, \Re)$ is generated by the symbols $e_{i j}(u)$, where $i$ and $j$ are distinct integers between 1 and $n$ and $u \in \Re$, subject to the relations

Received by the editors January 5, 1976 and, in revised form, March 18, 1976.

AMS (MOS) subject classifications (1970). Primary 20H25; Secondary 16A54.

1 This paper was originally part of the author's doctoral dissertation at the University of California, Los Angeles, under the direction of Robert Steinberg. 
(A)

$$
\begin{aligned}
e_{i j}(u) e_{i j}(v) & =e_{i j}(u+v), \\
\left(e_{i j}(u), e_{k l}(v)\right) & = \begin{cases}1 & \text { if } j \neq k, i \neq l, \\
e_{i l}(u v) & \text { if } j=k, i \neq l, \\
e_{k j}(-v u) & \text { if } j \neq k, i=l .\end{cases}
\end{aligned}
$$

Let $m_{i j}(u), d_{i j}(u), a_{1}(u, v)$ be as in (1), (2), and (3).

$$
a_{1}(u, v)=d_{1 j}(u) d_{1 j}(v) d_{1 j}(v u)^{-1}
$$

if $j \neq 1$ and

$$
a_{1}(u, v)=d_{i j}(u) d_{i j}(v) d_{i j}(v u)^{-1} d_{1 i}((u, v))
$$

if $1, i, j$ are distinct.

$$
\prod_{j=1}^{s} a_{1}\left(u_{j}, v_{j}\right)^{\varepsilon_{j}}=1 \quad \text { if } \prod_{j=1}^{s}\left(u_{j}, v_{j}\right)^{\varepsilon_{j}}=1, \varepsilon_{j}= \pm 1
$$

Proof. Certainly (A), (B), (C), (D) hold in SL( $n, \Re)$.

Now let $\operatorname{St}(n, \Re)$ be the Steinberg group generated by the symbols $x_{i j}(u)$, where $i$ and $j$ are distinct integers between 1 and $n$ and $u \in R$, subject to the relations obtained by replacing the $e_{i j}(u)$ by $x_{i j}(u)$ in (A), (B). For $u, v \in \mathscr{R}^{*}$, we define elements $w_{i j}(u), h_{i j}(u)$, and $b(u, v)$ of $\mathrm{St}(n, \Re)$ as

$$
\begin{aligned}
w_{i j}(u) & =x_{i j}(u) x_{j i}\left(-u^{-1}\right) x_{i j}(u), \\
h_{i j}(u) & =w_{i j}(u) w_{i j}(-1), \\
b(u, v) & =h_{12}(u) h_{12}(v) h_{12}(v u)^{-1} .
\end{aligned}
$$

If $i$ and $j$ are distinct between 1 and $n$ and $u \in \Re$ we consider symbols $\bar{x}_{i j}(u)$. Define $\bar{w}_{i j}(u), \bar{h}_{i j}(u), \bar{b}(u, v)$ in terms of the symbols $\bar{x}_{i j}(u)$ as we defined $w_{i j}(u), h_{i j}(u), b(u, v)$ in terms of $x_{i j}(u)$. Let $G$ be the group generated by the symbols $\bar{x}_{i j}(u)$ subject to relations $(\overline{\mathrm{A}}),(\overline{\mathrm{B}}),(\overline{\mathrm{C}}),(\overline{\mathrm{D}})$, obtained from (A), (B), (C), (D) by replacing $e_{i j}(u), m_{i j}(u), d_{i j}(u), a_{1}(u, v)$ by $\bar{x}_{i j}(u), \bar{w}_{i j}(u), \bar{h}_{i j}(u)$, $\bar{b}(u, v)$, respectively.

Therefore we have epimorphisms $\operatorname{St}(n, \Re) \stackrel{\varphi}{\longrightarrow} G \stackrel{\alpha}{\longrightarrow} \operatorname{SL}(n, \Re)$ defined by $\varphi\left(x_{i j}(u)\right)=\bar{x}_{i j}(u)$ and $\alpha\left(\bar{x}_{i j}(u)\right)=e_{i j}(u)$. We wish to prove $\alpha$ is an isomorphism.

Let $W$ be the subgroup of $\operatorname{St}(n, \Re)$ generated by the $w_{i j}(u), H$ the subgroup generated by the $h_{i j}(u)$, and $B$ the subgroup generated by the $b(u, v)$. Let $\bar{W}, \bar{H}$, and $\bar{B}$ be the corresponding subgroups of $G$.

Lemma 1. The kernel of $\alpha \varphi$ is contained in H. Hence the kernel of $\alpha$ is contained in $\bar{H}$. 
Proof. By [1, p. 78, Theorem 9.12], $\operatorname{ker}(\alpha \phi) \subset W$, and hence is equal to the kernel of $\left.\alpha \varphi\right|_{W}: W \rightarrow \operatorname{SL}(n, \Re)$. As in the proof of [1, p. 77, Theorem 9.11] we have $\operatorname{ker}(\alpha \varphi) \subset H$. The second statement follows immediately from the first.

LEMMA 2. Every element of $\bar{H}$ can be written in the form $\bar{b} \prod_{i=1}^{n-1} \bar{h}_{i, i+1}\left(u_{i}\right)$ where $\bar{b} \in \bar{B}$.

Proof. Let $H_{i}$ be the subgroup of $H$ generated by the $h_{i, i+1}(u)$. Then by [1, p. 72, Corollary 9.4] $H_{i}$ is normalized by all $h_{k l}(u)$, so $H_{1} H_{2} \cdots H_{n-1}$ is a subgroup. We will now show $H=H_{1} H_{2} \cdots H_{n-1}$, that is, that $H_{1} \cdots H_{n-1}$ contains all $h_{k l}(u), k<l$. If $l=k+1$, it is clear, and we proceed by induction on $l-k$. Choose $p$ so that $k<p<l$. Then by [1, p. 76, Lemma 9.10] $h_{k l}(u)=h_{p l}(u) h_{k p}(u)$, and each of these is in $H_{1} H_{2} \cdots H_{n-1}$ by induction. Therefore $H=H_{1} H_{2} \cdots H_{n-1}$. Now if $\bar{H}_{i}$ is the subgroup of $\bar{H}$ generated by the $\bar{h}_{i, i+1}(u)$, we have that $\bar{H}=\bar{H}_{1} \bar{H}_{2} \cdots \bar{H}_{n-1}$.

By a similar argument, $\bar{H}_{1} \bar{H}_{2} \cdots \bar{H}_{i-1}$ is the subgroup generated by all $\bar{h}_{k l}(u), k<l \leqslant i$.

If $\bar{h} \in \bar{H}, \bar{h}=\bar{h}_{1} \bar{h}_{2} \cdots \bar{h}_{n-1}$ where $\bar{h}_{i} \in \bar{H}_{i}$, since $\bar{H}=\bar{H}_{1} \bar{H}_{2} \cdots \bar{H}_{n-1}$.

Claim. If $i \neq 1, \bar{h}_{i}=\bar{h}^{\prime} \bar{h}_{i, i+1}(u)$ for some $u \in \mathscr{R}^{*}$ and some $\bar{h}^{\prime}$ $\in \bar{H}_{1} \bar{H}_{2} \cdots \bar{H}_{i-1}$.

Suppose

$$
\bar{h}_{i}=\bar{h}_{i, i+1}\left(u_{1}\right)^{\varepsilon_{1}} \bar{h}_{i, i+1}\left(u_{2}\right)^{\varepsilon_{2}} \cdots \bar{h}_{i, i+1}\left(u_{r}\right)^{\varepsilon_{r}}, \quad r \geqslant 1, \varepsilon_{i}= \pm 1 .
$$

Since $\bar{h}_{i, i+1}(u)^{-1}=\bar{b}\left(u^{-1}, u\right)^{-1} \bar{h}_{i, i+1}\left(u^{-1}\right)$, by $(\overline{\mathrm{C}})$, we may assume $\varepsilon_{1}=1, r$ $>1$, and

$$
h_{i}=\bar{h}_{i, i+1}\left(u_{1}\right) \bar{h}_{i, i+1}\left(u_{2}\right)^{\varepsilon_{2}} \cdots \bar{h}_{i, i+1}\left(u_{r}\right)^{\varepsilon_{r}}
$$

If $\varepsilon_{2}=1$ we have

$$
\bar{h}_{i}=\bar{b}\left(u_{1}, u_{2}\right) \bar{h}_{1 i}\left(\left(u_{1}, u_{2}\right)\right)^{-1} \bar{h}_{i, i+1}\left(u_{2} u_{1}\right) \bar{h}_{i, i+1}\left(u_{3}\right)^{\varepsilon_{3}} \cdots \bar{h}_{i, i+1}\left(u_{r}\right)^{\varepsilon_{r}}
$$

by $(\overline{\mathrm{C}})$, and $\bar{b}\left(u_{1}, u_{2}\right) \bar{h}_{1 i}\left(\left(u_{1}, u_{2}\right)\right)^{-1} \in \bar{H}_{1} \bar{H}_{2} \cdots \bar{H}_{i-1}$. If $\varepsilon_{2}=-1$ we have

$$
\begin{gathered}
\bar{h}_{i, i+1}\left(u_{1}\right) \bar{h}_{i, i+1}\left(u_{2}\right)^{-1}=\left(\bar{h}_{i, i+1}\left(u_{2}\right) \bar{h}_{i, i+1}\left(u_{1}\right)^{-1}\right)^{-1} \\
=\left[\bar{h}_{i, i+1}\left(u_{2}^{-1} u_{1}\right)^{-1} \bar{b}\left(u_{2}^{-1} u_{1}, u_{2}\right) \bar{h}_{1 i}\left(\left(u_{2}^{-1}, u_{1}\right)\right)^{-1}\right]^{-1} \\
=\bar{h}_{1 i}\left(\left(u_{2}^{-1}, u_{1}\right)\right) \bar{b}\left(u_{2}^{-1} u_{1}, u_{2}\right)^{-1} \bar{h}_{i, i+1}\left(u_{2}^{-1} u_{1}\right)
\end{gathered}
$$

by $(\overline{\mathbf{C}})$. Hence in this case

$$
\bar{h}_{i}=\bar{h}_{1 i}\left(\left(u_{2}^{-1}, u_{1}\right)\right) \bar{b}\left(u_{2}^{-1} u_{1}, u_{2}\right)^{-1} \bar{h}_{i, i+1}\left(u_{2}^{-1} u_{1}\right) \bar{h}_{i, i+1}\left(u_{3}\right)^{\varepsilon_{3}} \cdots \bar{h}_{i, i+1}\left(u_{r}\right)^{\varepsilon_{r}},
$$

and $\bar{h}_{1 i}\left(\left(u_{2}^{-1}, u_{1}\right)\right) \bar{b}\left(u_{2}^{-1} u_{1}, u_{2}\right)^{-1} \in \bar{H}_{1} \bar{H}_{2} \cdots \bar{H}_{i-1}$. 
By induction on $r$ and since $\bar{H}_{1} \bar{H}_{2} \cdots \bar{H}_{i-1}$ is a subgroup we have that $\bar{h}_{i}=\bar{h} \bar{h}_{i, i+1}(u)$ as desired.

By the claim we get

$$
\bar{h}=\bar{h}_{1}^{\prime} \bar{h}_{23}\left(u_{3}\right) \cdots \bar{h}_{n-1, n}\left(u_{n}\right)
$$

where $\bar{h}_{1}^{\prime} \in H_{1}$. Using induction and the definition of $\bar{b}(u, v)$, we have $\bar{h}_{1}^{\prime}=\overline{b h}_{12}\left(u_{2}\right)$ so that

$$
\bar{h}=\overline{b h}_{12}\left(u_{2}\right) \cdots \bar{h}_{n-1, n}\left(u_{n}\right) .
$$

We will now complete the proof of the Theorem by showing that $\alpha$ is an isomorphism. Let $x \in \operatorname{ker} \alpha$. By Lemma $1 x \in \bar{H}$, so by Lemma 2

$$
x=\bar{b} \prod_{i=1}^{n-1} \bar{h}_{i, i+1}\left(u_{i}\right)
$$

Applying $\alpha$ we obtain

$$
1=a \prod_{i=1}^{n-1} d_{i, i+1}\left(u_{i}\right)
$$

where $a \in A$, the subgroup generated by all $a_{1}(u, v), u, v \in \Re^{*}$. Therefore if $i \geqslant 2, u_{i-1}^{-1} u_{i}=1$, and $u_{n-1}^{-1}=1$, which implies $u_{i}=1$ for $i=1,2, \ldots$, $n-1$. Hence

$$
x=\bar{b}=\prod_{j=1}^{s} \bar{b}\left(u_{j}, v_{j}\right)^{\varepsilon_{j}}
$$

for some $u_{j}, v_{j} \in \mathscr{R}^{*}, \varepsilon_{j}= \pm 1$. Applying $\alpha$ to this expression we obtain

$$
1=\prod_{j=1}^{s} a_{1}\left(u_{j}, v_{j}\right)^{\varepsilon_{j}}
$$

which implies $\prod_{j=1}^{s}\left(u_{j}, v_{j}\right)^{\varepsilon_{j}}=1$, and $x=\bar{b}=1$ by $(\overline{\mathrm{D}})$. Therefore $\alpha$ is an isomorphism.

\section{BIBLIOGRAPHY}

1. J. Milnor, Introduction to algebraic K-theory, Ann. of Math. Studies, no. 72, Princeton Univ. Press, Princeton, N.J.; Univ. of Tokyo Press, Tokyo, 1971. MR 50 \# 2304.

Department of Mathematics, University of Utah, Salt Lake City, Utah 84112 